\title{
Solvent Effects on the Two-Photon Absorption of Distyrylbenzene Chromophores
}

Han Young Woo, Bin Liu, Bernhard Kohler, Dmitry Korystov, Alexander Mikhailovsky and Guillermo C. Bazan*

Mitsubishi Chemical Center for Advanced Materials, Department of Materials, Institute for Polymers and Organic Solids, University of California, Santa Barbara, California 93106

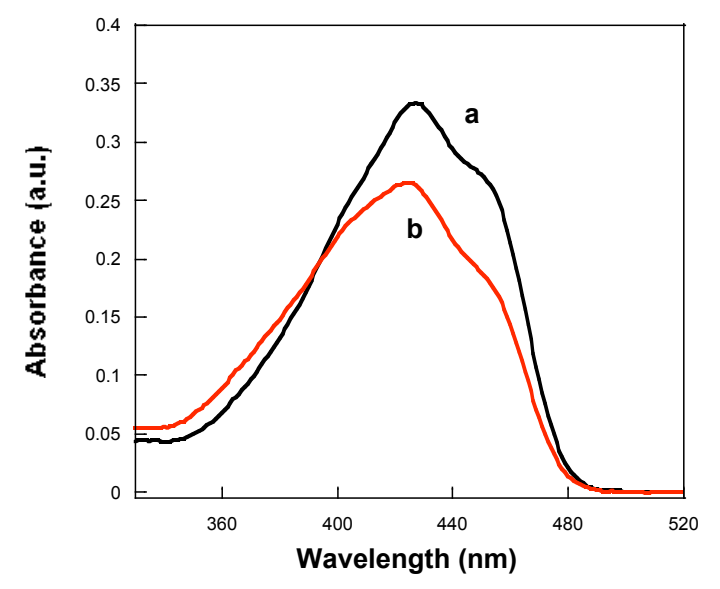

Figure S1. UV-vis spectrum change $(\mathrm{a} \rightarrow \mathrm{b})$ of $\mathbf{1 1} \mathbf{N}$ in toluene after being left on a lab bench for 30 minutes. The maximum absorbance decreases approximately $20 \%$ and the relative intensity of the blue region increases, leading to a change of the band shape. 


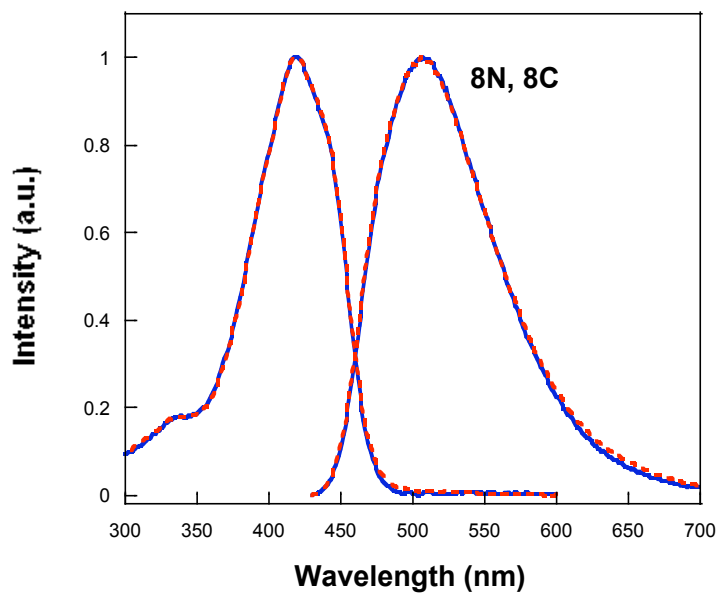

Figure S2. Normalized absorption and PL spectra of $\mathbf{8 N}$ (red dotted line) and $\mathbf{8 C}$ (blue solid line) in DMSO.

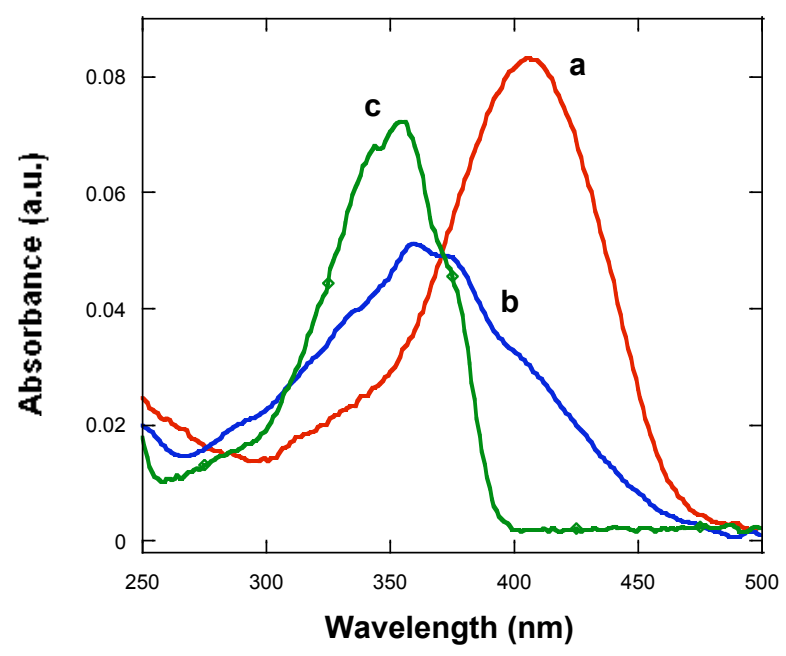

Figure S3. Absorption spectra of $\mathbf{8 C}(\mathrm{a}), \mathbf{8} \mathrm{CH}^{+}$(b) and $\mathbf{8 C H}_{2}{ }^{2+}$ (c) in water. The extinction coefficient $\left(\varepsilon_{8 \mathrm{CH}}^{(i)}(\lambda)\right)$ of mono-protonated component $\left(\mathbf{8} \mathbf{C H}^{+}\right)$was extracted by deconvolution using the general equilibrium analysis technique (eq. 6 and S.13 in ref 1) at intermediate $[\mathrm{HCl}]$. 


\section{TPA Spectra Measurements}

The TPA spectra of the chromophores were obtained by the two-photon induced fluorescence (TPIF) measurement technique by using femto-second laser pulses as described in the literature. ${ }^{2,3}$ The samples are excited via TPA by directing a tightly collimated, high intensity laser beam onto the sample. The emission from the sample was collected at a $90^{\circ}$ angle by a high numerical aperture lens and directed to a spectrometer's entrance slit. To avoid internal filter effects, the excited volume was located near the cell wall on the collection optics side. This configuration minimizes the fluorescence path inside the sample cell and thus reduces self-absorption. Additionally, the TPIF intensity was measured at wavelengths removed from the absorption of the sample, i.e. in the transparent region. The radiation dispersed by the spectrometer was detected by a photomultiplier tube operating in the photon counting mode (Hamamatsu H8259-02) connected to a computer counter board (Hamamatsu M8784). Excitation pulses with typical duration 90 fs and energy $\sim 6 \mathrm{~nJ}$ were produced by the mode-locked Ti:Sapphire laser (Spectraphysics Tsunami) with repetition rate of $82 \mathrm{MHz}$. The laser wavelength was tuned continuously within the spectral range of 700-900 nm. A neutral density filter wheel was used to attenuate the energy of the laser pulses down to the desirable level. The molar concentration of the compounds was determined from optical absorption spectra, using the molar absorptivity values supplied by the compound manufacturer or obtained from volumetric measurements. In all measurements, the concentration of the sample was adjusted around $10^{-5} \mathrm{M}$, to avoid emission self-quenching. Fluorescence quantum yields were measured relative to fluorescein and 9,10-diphenylanthracene and verified using the referenceless technique described previously. ${ }^{4}$ It is worth mentioning that both the absorption and the emission spectra of the samples were monitored during the entire series of measurements. After being properly degassed, our samples did not exhibit any sign of degradation within the timescale of the experiment.

As the reference materials for TPA measurements in the spectral range of 700-900 nm, we used three laser dyes: Fluorescein (Acros Inc.), Coumarin 503 (aka Coumarin 307, Exciton Inc.) and Rhodamine 610 (aka Rhodamine B, Exciton Inc.). The TPA values relative to the standard fluorescein were verified using the other references of coumarin and rhodamine. The measured $\delta$ values using three different references are in good agreement in the experimental error range $(\sim 15 \%)$. Fluorescein was prepared in water $(\mathrm{pH} \approx 11)$ and 
coumarin and rhodamine were dissolved in methanol at $\sim 10^{-5} \mathrm{M}$.

According to reference 2, the spectrally integrated intensity of the fluorescence generated via TPA process can be determined by the following expression.

$$
I=A \eta \int_{-\infty}^{\infty} \frac{I_{0}^{2}(t) \delta c l}{1+I_{0}(t) \delta c l} d t
$$

Here, $\delta$ is the TPA cross section and $l$ corresponds to the interaction length. The signal intensity collected by detector is denoted as $I$ and $\eta$ is the fluorescence quantum yield. The number density of the molecules in solution is denoted as $c$. Factor $A$ includes all constant parameters specific to the given experimental setup (geometric factors, spectrometer and detector efficiencies, etc.). Function $I_{0}(t)$ describes the temporal profile of the excitation pulse. In case of the weak excitation regime, one can assume that $I_{0}(t) \delta n l \ll 1$. Then expression (S1) takes the form,

$$
I=A \eta \delta c l \int_{-\infty}^{\infty} I_{0}^{2}(t) d t=B \eta \delta c l P^{2}
$$

$P$ and $B$ are excitation light power and a constant, respectively. Since $B$ is difficult to determine, we employed relative measurements technique using a reference with the known TPA spectrum. In this case, the ratio of the integrated PL intensities for the reference and the studied sample can be expressed as

$$
\frac{I}{I_{r e f}}=\frac{\eta \delta c}{\eta_{r e f} \delta_{r e f} c_{r e f}} \frac{P^{2}}{P_{r e f}^{2}} K
$$

Here, the index ref denotes values related to the reference measurements. $\delta_{r e f}$ means the TPA cross section of the reference solution. Also, we assume that all experimental parameters are identical during the whole series of measurements, except the power of the pump radiation $(P)$ and sample specific parameters, $\eta$ and $c . K$ is a correction factor taking into account the difference in refractive indices of the solvents of the studied and the reference material. It depends on the excitation beam geometry. In our case of nearly collimated beams, $K=n_{r e f}^{2} / n^{2}$. The schematic experimental setup for TPA measurement is 
described in Figure S4.

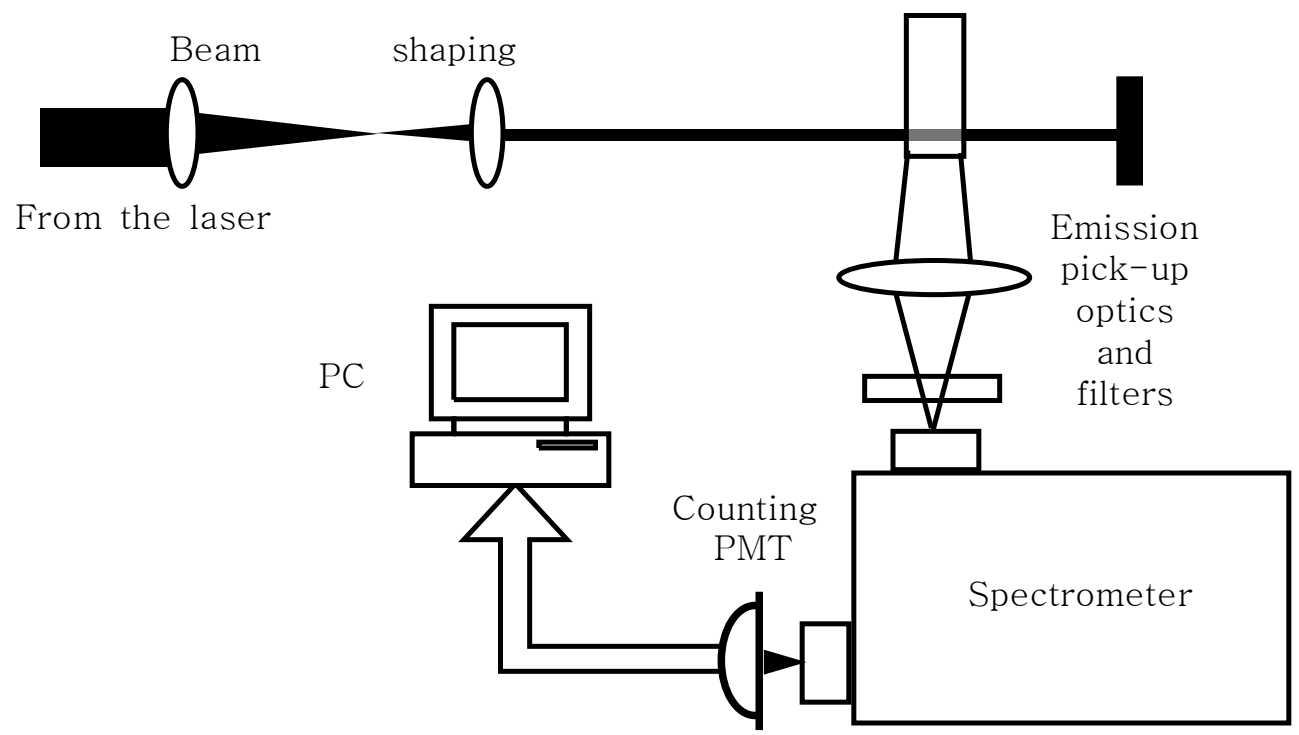

Figure S4. Experimental setup for the TPA cross section measurements.

TPIF spectra of the standard and sample were recorded at the same excitation wavelength in low excitation power regime to remove the contributions from other nonlinear effects such as excited state absorption or three-photon absorption, etc. It was also verified by measuring the quadratic dependence of the TPIF signal on the laser pump intensity, indicating that the signal originated from a pure nonlinear optical process. 


\section{Aggregation of $8 \mathrm{C}$ in water}

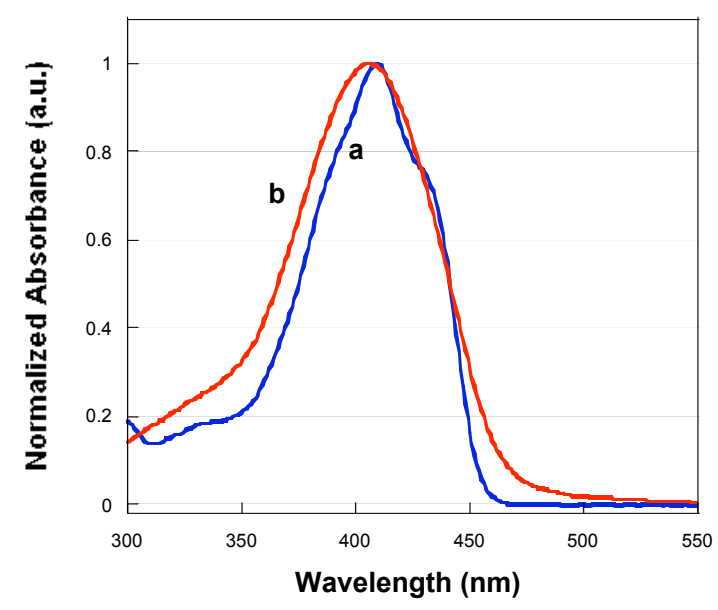

Figure S5. UV/vis spectra of $\mathbf{8 N}$ in toluene (a) and $\mathbf{8 C}$ in water (b). $[\mathbf{8 N}$ or $\mathbf{8 C}]=10^{-5} \mathrm{M}$
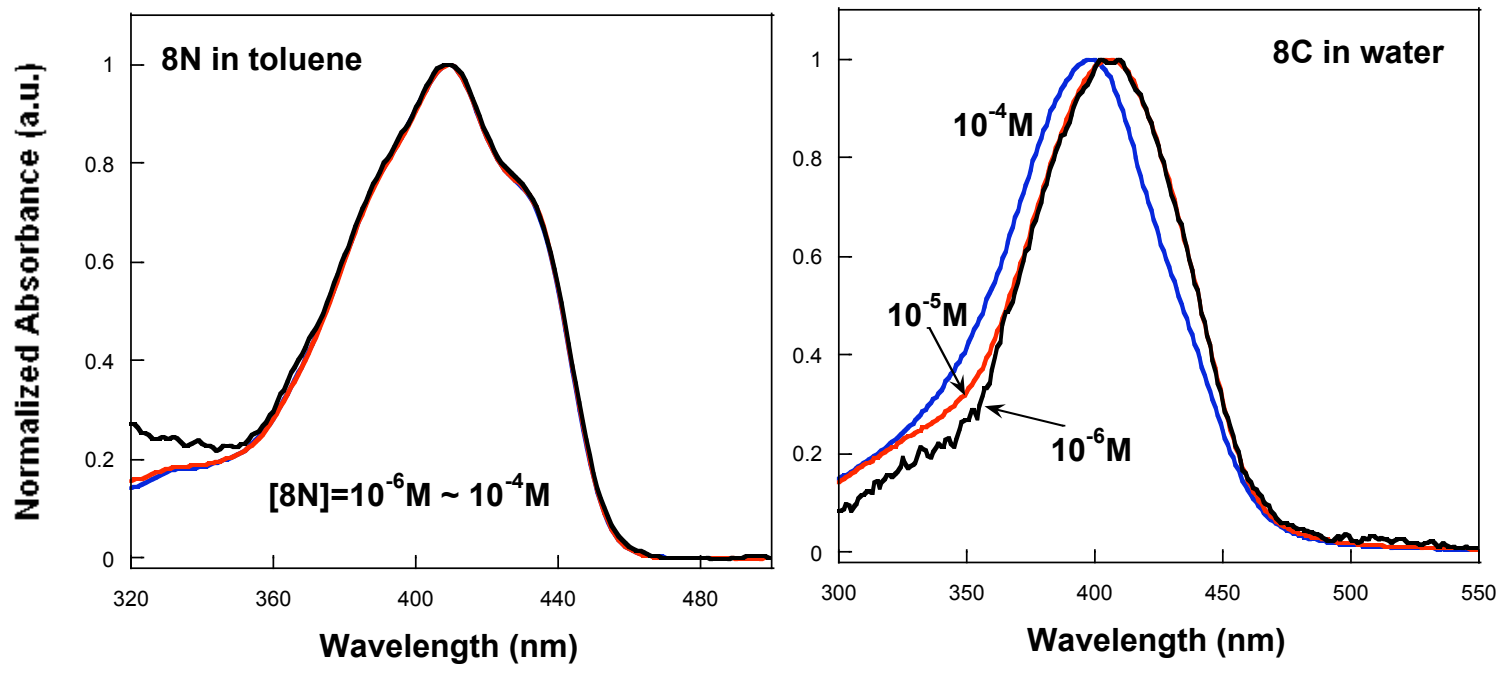

Figure S6. Normalized absorption spectra of $\mathbf{8 N}$ in toluene and $\mathbf{8 C}$ in water at different concentrations. $[\mathbf{8 N}$ or $\mathbf{8 C}]=10^{-6} \sim 10^{-5} \mathrm{M}$. 


\section{Complete Ref. 1(b).}

Cumpston, B. H.; Anathavel, S. P.; Barlow, S.; Dyer, D. L.; Ehrlich, J. E.; Erskine, L. L.;

Heikal, A. A.; Kuebler, S. M.; Lee, I.-Y.; McCord-Maughon, D.; Qin, J.; Röckel, H.; Rumi, M.; Wu, X.-L.; Marder, S. R.; Perry, J. W. Nature 1999, 398, 51.

\section{Complete Ref. 11.}

Albota, M.; Beljonne, D.; Brédas, J.-L.; Ehrlich, J. E.; Fu, J.-Y.; Heikal, A. A.; Hess, S. E.;

Kogej, T.; Levin, M. D.; Marder, S. R.; McCord-Maughon, D.; Perry, J. W.; Röckel, H.;

Rumi, M.; Subramaniam, G.; Webb, W. W.; Wu, X.-L.; Xu, C. Science 1998, 281, 1653.

\section{References}

1 Pond, S. J. K.; Tsutsumi, O.; Rumi, M.; Kwon, O.; Zojer, E.; Brédas, J.-L.; Marder, S. R.; Perry, J. W. J. Am. Chem. Soc. 2004, 126, 9291.

2 Xu, C.; Webb, W. W. J. Opt. Soc. Am. B 1996, 13, 481.

3 Rumi, M.; Ehrlich, J. E.; Heikal, A. A.; Perry, J. W.; Barlow, S.; Hu, Z.; McCordMaughon, D.; Parker, T. C.; Röckel, H.; Thayumanavan, S.; Marder, S. R.; Beljonne, D.; Brédas, J.-L. J. Am. Chem. Soc. 2000, 122, 9500.

${ }^{4}$ Greenham, N. C.; Samuel, I. D.W.; Hayes, G. R.; Phillips, R. T.; Kessener, Y. A. R. R.; Moratti, S. C.; Holmes, A. B.; Friend, R. H. Chem. Phys. Lett. 1995, 241, 89. 